\title{
The Population Prediction based on Grey Model and BP Neural Networks
}

\author{
Meng Liu a, Yonglin Pang and Wei Xiang ${ }^{b, *}$ \\ College of Electric and Information Engineering, Southwest University for Nationalities, 610041, \\ Chengdu, china \\ a1792567679@qq.com, b3730544@qq.com \\ Corresponding author: Wei Xiang
}

Keywords: Population prediction; Grey model; BP neural network; Combination prediction model; MATLAB.

\begin{abstract}
In this paper, the methods of population prediction are analyzed and studied. Considering the limitation of using the grey model and BP neural network, we established a new combination prediction model based on the two intelligent algorithms. First, the grey model and BP neural network were used to do data simulation on MATLAB. Then, a new combination forecasting model is obtained by using variance distribution. The result showed that the combination model can give full play to the advantages of single model as well as weaken its disadvantages and it is more ideal than the single model.
\end{abstract}

\section{Introduction}

Population has been one of the most striking features of China's national condition. The huge population has made many impacts on social economic development, although it provides a wealth of labor, at the same time it has forced economic development, social progress, resource utilization and environmental protection to face heavy pressure[1]. Scientific and accurate prediction of population is an important procedure in the development of economy and society, and it has important practical significance to research economic resources and social problems in the next period of time, but also can provide a theoretical basis for the government to formulate relevant policies.

\section{The Overview of Single Prediction Methods}

Prediction is a science of predicting future events, which is extrapolated to the future by collecting historical data. When we predict, three is no ways that is relatively valid without errors. The traditional research methods, such as moving average method, exponential smoothing method, linear regression equation time and series method are commonly used. After that, artificial intelligence algorithms which includes artificial neural network, fuzzy theory and grey theory ${ }^{[2]}$ emerged, and in this passage we use artificial neural networks and grey theory as prediction methods.

\subsection{Gray Prediction}

The Grey prediction method[3] is a way that it firstly mainly generates a ordered sequence by adding or subtracting original data consecutively, and then establish a differential equation with generated-data at the same time by solving the differential generate the prediction model, and finally recover the arithmetic results when finish the prediction. To solve problems which are uncertain with less data, it has a unique advantage-the data is small, the modeling process is simple and the prediction accuracy is high- In so many grey models, the first order linear differential equation model GM $(1,1)$ is the most common one used. The steps of $\operatorname{GM}(1,1)$ prediction model are as follows:

The original sequence

$$
x^{(0)}=\left\{x^{(0)}(1), x^{(0)}(2), \ldots, x^{(0)}(n)\right\}
$$

$n$ is the number of data, and make a cumulation to generate the following equation

$$
x^{(1)}=\left\{x^{(1)}(1), x^{(1)}(2), \ldots, x^{(1)}(n)\right\}
$$


In the equation above

$$
x^{(1)}=\sum_{j=1}^{i} x^{(0)}(j)(i=1,2, \ldots, n)
$$

Establishing first order linear differential equation

$$
\frac{d x^{(1)}}{d_{t}}+a x^{(1)}=u
$$

In the equation, $a$ is the coefficient of development and $u$ is the grey function. Use least squares method to solve the parameters $a$ and $u$.

$$
\hat{a}=\left\{\begin{array}{l}
a \\
u
\end{array}\right\}=\left(B^{T} B\right)^{-1} B^{T} Y_{n}
$$

Generate the $\mathrm{GM}(1,1)$ model

$$
\hat{x}^{(1)}(k+1)=\left[x^{0}(1)-\frac{u}{a}\right] e^{-a k}+\frac{u}{a},(k=0,1,2, \ldots)
$$

Recover the prediction results

$$
\hat{x}^{(0)}(k+1)=\hat{x}^{(1)}(k+1)-\hat{x}^{(1)}(k)
$$

\subsection{BP Neural Networks}

BP (back propagation, BP) Neural Network ${ }^{[4]}$ with the learning and memory function like the human brain can be used for prediction and function optimization by simulating the human brain neural processing of information. BP Neural Network has three or more than three layers of neural network, including input layer, hidden layer and output layer.

The middle of input and output of BP Neural Networks is a kind of highly nonlinear mapping relation, and we can simulate a steady neural mapping only by using variables and results. A complete BP Neural Networks is composed of two procedures involving positive transmission of information and negative transmission of information. First, the BP algorithm carries forward the input signal, the input sample first enters the input layer of the network, and then through the analysis and calculation of the hidden layer in the middle, it enters the output layer to get the result of the sample training output. If there is an error between the final output of the network and the expected output of the network, the error will be transmitted back. The error back-propagation learning algorithm will first error according to a certain arrangement into the corresponding form, by the spread of hidden layer to the input layer, and according to the error distribution to all neurons in each layer, so as to get the error signal of every layer, the error signal can be used as the basis for correction of neuron weights. This weight correction process is the network learning and training process. This process is iterated, and finally the error signal is within the allowable range.

\section{MATLAB Simulation}

The data in this paper are from China Statistical Yearbook in 1995-2004. Use GM $(1,1)$ and BP Neural Networks on the MATLAB to do data simulation ${ }^{[5]}$. Accumulate the relative error according to following formula. The results are shown in Table 1.

$$
\text { Relative error }=\frac{\text { True value }- \text { Predictive value }}{\text { True value }} \times 100 \%
$$

From the Table 1, the maximum relative error of these two single prediction models is not more than $0.2 \%$. The maximum relative error and the average relative error of GM is greater than BPNN, which indicates that the grey prediction system's forecast effect is not very ideal for volatility system and the forecast accuracy rate will gradually decrease with time extrapolation. Also, BP Neural Network does not have a model test on the latter, which may be accidental. Moreover, it often needs a lot of data when training the network. In order to make full use of the effective information of a single prediction, overcome the shortcomings of a single model, reduce the randomness of the forecast, and improve the accuracy of prediction, we introduce the combination forecasting model. 
Table 1. Prediction and relative error of the single model

\begin{tabular}{|c|c|c|c|c|c|}
\hline \multirow[b]{2}{*}{ Year } & \multirow[b]{2}{*}{ True Value } & \multicolumn{2}{|c|}{$\mathrm{GM}(1,1)$} & \multicolumn{2}{|c|}{ BP Neural Networks } \\
\hline & & $\begin{array}{l}\text { Predictive } \\
\text { Value }\end{array}$ & Relative Error & $\begin{array}{l}\text { Predictive } \\
\text { Value }\end{array}$ & Relative Error \\
\hline 1995 & 121121 & 121121.0 & $0.00 \%$ & 121121.0 & $0.00 \%$ \\
\hline 1996 & 122389 & 122787.2 & $0.3254 \%$ & 122067.3 & $0.016 \%$ \\
\hline 1997 & 123626 & 123702.0 & $0.0615 \%$ & 123724.8 & $0.035 \%$ \\
\hline 1998 & 124761 & 124623.6 & $0.1102 \%$ & 124757.0 & $0.029 \%$ \\
\hline 1999 & 125786 & 125552.0 & $0.1860 \%$ & 125795.2 & $0.0005 \%$ \\
\hline 2000 & 126743 & 126487.4 & $0.2017 \%$ & 126727.0 & $0.015 \%$ \\
\hline 2001 & 127627 & 127429.7 & $0.1546 \%$ & 127650.9 & $0.022 \%$ \\
\hline 2002 & 128453 & 128379.0 & $0.0576 \%$ & 128420.1 & $0.013 \%$ \\
\hline 2003 & 129227 & 129335.5 & $0.0839 \%$ & 129348.4 & $0.014 \%$ \\
\hline 2004 & 129988 & 130299.0 & $0.2393 \%$ & 129845.4 & $0.084 \%$ \\
\hline \multicolumn{2}{|c|}{$\begin{array}{l}\text { Maximum Relative } \\
\text { Error }\end{array}$} & \multicolumn{2}{|c|}{$0.325368 \%$} & \multicolumn{2}{|c|}{$0.262818 \%$} \\
\hline \multicolumn{2}{|c|}{ Average Relative Error } & \multicolumn{2}{|c|}{$0.142009 \%$} & \multicolumn{2}{|c|}{$0.061386 \%$} \\
\hline
\end{tabular}

\section{Combination Prediction Model}

Considering the limitation of the single prediction method and in order to obtain more satisfied results, we can use the information provided by the various forecasting methods to establish a new combination forecasting model. Supposing the variance of the predicted value of the grey prediction and BP neural network respectively is $\alpha_{1}$ and $\alpha_{2}$, and $k_{i}$ is the weighted coefficients of the $i$ prediction methods, $X_{i}$ is the predictive value of the $i$ prediction method, $X_{c}$ is the combined predictive value. The weighted coefficients and the predicted values of the combined model respectively are

$$
k_{i}=\frac{\alpha-\alpha_{i}}{\alpha}, \quad X_{c}=\sum_{i=1}^{2} \alpha_{i} X_{i}, \quad(i=1,2)
$$

According to the variance calculation method, we get the weights of the grey model and BP neural network and the predictive results are shown in Table 2.

$$
X_{c}=0.506472 X_{1}+0.493528 X_{2}
$$

Table 2. Predictive value and relative error of combined forecasting model

\begin{tabular}{|c|c|c|c|}
\hline \multirow{2}{*}{ Year } & \multirow{2}{*}{ True Value } & \multicolumn{2}{|c|}{ Combined Forecasting Model } \\
\cline { 3 - 4 } & 121121 & Predictive Value & Relative Error \\
\hline 1995 & 122389 & 121121.0 & $0.00 \%$ \\
\hline 1996 & 123626 & 122431.9 & $0.035 \%$ \\
\hline 1997 & 124761 & 123713.2 & $0.0705 \%$ \\
\hline 1998 & 125786 & 124689.4 & $0.0573 \%$ \\
\hline 1999 & 126743 & 125672.0 & $0.0906 \%$ \\
\hline 2000 & 127627 & 126605.6 & $0.10838 \%$ \\
\hline 2001 & 128453 & 127538.9 & $0.06903 \%$ \\
\hline 2002 & 129227 & 128399.3 & $0.04177 \%$ \\
\hline 2003 & 129988 & 129341.9 & $0.0889 \%$ \\
\hline 2004 & 130075.2 & $0.0671 \%$ \\
\hline \multicolumn{2}{|c|}{ Maximum Relative Error } & $0.10838 \%$ & \\
\hline \multicolumn{2}{|c|}{ Average Relative Error } & $0.06287 \%$ \\
\hline
\end{tabular}

Compared with the single model, it can be seen that the combined forecasting model has a more accurate prediction effect and the maximum relative error is $0.11 \%$ smaller than that of the single model, and the average relative error is $0.06 \%$ less than others. 


\section{Model Vaildation And Comparison}

In order to further verify the correctness of the combined forecasting model, and compared with the superiority of the single model, we validate the 2005-2009 population. Results are shown in Table 3.

Table 3. Verification and comparison of three predictive methods

\begin{tabular}{|c|c|c|c|c|c|c|c|}
\hline \multirow{2}{*}{ Year } & \multirow{2}{*}{$\begin{array}{c}\text { True } \\
\text { Value }\end{array}$} & \multicolumn{2}{|c|}{ GM (1,1) } & \multicolumn{2}{c|}{ BP Neural Networks } & \multicolumn{2}{c|}{$\begin{array}{c}\text { Combined Forecasting } \\
\text { Model }\end{array}$} \\
\cline { 3 - 8 } & & $\begin{array}{c}\text { Predictive } \\
\text { Value }\end{array}$ & $\begin{array}{c}\text { Relative } \\
\text { Error }\end{array}$ & $\begin{array}{c}\text { Predictive } \\
\text { Value }\end{array}$ & $\begin{array}{c}\text { Relative } \\
\text { Error }\end{array}$ & $\begin{array}{c}\text { Predictive } \\
\text { Value }\end{array}$ & $\begin{array}{c}\text { Relative } \\
\text { Error }\end{array}$ \\
\hline 2005 & 130756 & 131269.7 & $0.3929 \%$ & 132663.8 & $1.4590 \%$ & 131960.2 & $0.9191 \%$ \\
\hline 2006 & 131448 & 132247.7 & $0.6084 \%$ & 132844.8 & $1.0626 \%$ & 132540,3 & $0.8326 \%$ \\
\hline 2007 & 132129 & 133232.9 & $0.8355 \%$ & 132808.7 & $0.5144 \%$ & 133020,7 & $0.6770 \%$ \\
\hline 2008 & 132802 & 134225.5 & $1.0719 \%$ & 132653.4 & $0.1119 \%$ & 133450,5 & $0.4877 \%$ \\
\hline 2009 & 133450 & 135225.5 & $1.3304 \%$ & 132566.6 & $0.6619 \%$ & 133910,2 & $0.3471 \%$ \\
\hline \multicolumn{2}{|c|}{$\begin{array}{c}\text { Maximum } \\
\text { Relative Error }\end{array}$} & $1.3304 \%$ & $1.4590 \%$ & $0.9191 \%$ \\
\hline $\begin{array}{c}\text { Average Relative } \\
\text { Error }\end{array}$ & \multicolumn{2}{|c|}{$0.8478 \%$} & $0.7620 \%$ & & $0.6527 \%$ \\
\hline
\end{tabular}

It can be seen from the diagram that the average relative error and the maximum relative error of the combined forecasting model are lower than that of the single model. The combination forecasting model is more accurate than the single grey prediction and BP neural network. In order to directly reflect the relative error of the three, we do the error comparison chart as shown in figure 1.

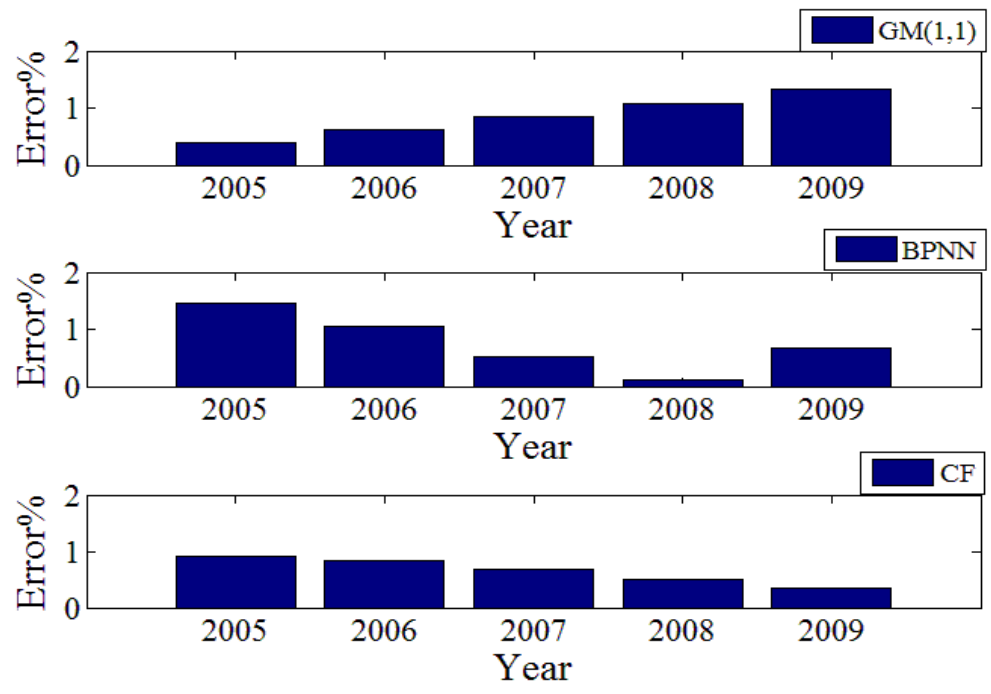

Figure 1. Relative error comparison of three methods in 2005-2009

It can be seen from the diagram that the error of grey prediction increases with time extrapolation, and the BP neural network has a large fluctuation and randomness. However, the combination model is more stable and the accuracy will not decrease with time extrapolation, so it overcomes the disadvantages of grey forecast and artificial neural network.

\section{Conclusion}

This paper focuses on the application of grey prediction and BP neural network in population prediction, at the same time puts forward a new combination forecasting model. Because the accuracy of the single grey prediction will decrease with the over time, so it is not suitable for the medium and 
long term prediction. For BP neural network, it has a lot of randomness, which leads to the uncertainty of the prediction accuracy. However, the combination forecasting model based on grey prediction and BP neural network in this paper can weaken the defects and absorb the advantages of both. To be specific, the prediction result and the error index are only changed in a small range in the prediction of many times, and the prediction accuracy is kept well.

Of course, in the actual situation there are a lot of factors such as policy changes and sudden events that are likely to cause substantial fluctuations in the general population. Therefore, the results of this paper are mainly used as a reference in theory, and it is also necessary for management decision makers to weigh the decision according to the actual situation.

In this paper, we only study one kind of combination, but there are many ways to combine the grey model with the artificial neural network. Accordingly, there are still some problems that need to be further studied in the future.

\section{Acknowledgments}

This work was partially supported by National Nature Science Foundation (61673016), SWUN Construction Projects for Graduate Degree Programs (2017XWD-S0805), Advance Research Program of Electronic Science and Technology National Program (2017YYGZS16), and Sichuan Youth Science and technology innovation research team (2017TD0028).

\section{References}

[1] Ping Yang, Analysis on the present situation and trend of population development in China [J], Technology entrepreneurship monthly, 2012, (08):167-169

[2] Qian Wang,Bing Jiang, A case based approach to water resources prediction [J], Technology and Industry,2008,(02):30-3

[3] Wenwei Tu, Qianbing Di,Jian Zhang, Application and comparison of several methods of population prediction: a case study of Luannan County, Hebei Province [J], Northern economy,2012,(11):34-36

[4] Jian Mao, Hongdong Zhao, Qianqian Yao,Development and application of artificial neural network [J],Electronic design engineering, 2011,(24):62-65

[5] Ping Li, Lingke Zeng, Anze Shui, Xueli Jin, Yanchun Liu, Hui Wang,Design of BP neural network prediction system based on MATLAB[J],Computer applications and software, 2008, (04):149-150+184. 